\title{
ARTICLE Theta-gamma coupling and ordering information: a stable brain-behavior relationship across cognitive tasks and clinical conditions
}

\author{
Heather Brooks ${ }^{1,2}$, Michelle S. Goodman ${ }^{1,2,3}$, Christopher R. Bowie ${ }^{1,3,4}$, Reza Zomorrodi ${ }^{1,3}$, Daniel M. Blumberger ${ }^{1,2,3,5}$, Meryl A. Butters ${ }^{6}$, \\ Zafiris J. Daskalakis ${ }^{1,2,3,5}$, Corinne E. Fischer ${ }^{5,7}$, Alastair Flint ${ }^{5,8}$, Nathan Herrmann ${ }^{5,9}$, Sanjeev Kumar ${ }^{1,2,3}$, Linda Mah ${ }^{5,10}$, \\ Benoit H. Mulsant ${ }^{1,2,5}$, Bruce G. Pollock ${ }^{1,2,5}$, Aristotle N. Voineskos ${ }^{1,2,5}$, Tarek K. Rajji ${ }^{1,2,3,5}$ and on behalf of the PACt-MD Study Group
}

\begin{abstract}
Ordering of information is a critical component that underlies several cognitive functions. Prefrontal theta-gamma coupling (TGC) is a neurophysiologic measure associated with ordering of information during the performance of a working memory task ( $\mathrm{N}$-back). Little is known about the relationship between TGC and ordering during other cognitive tasks or whether the relationship between TGC and ordering of information is independent of clinical condition. This study aimed to determine whether the relationship between TGC and ordering of information exists independent of a task and its timing, and whether this relationship is the same in different clinical conditions. A total of 311 participants were assessed using a neuropsychological battery that included the $\mathrm{N}$-back during which TGC was measured; two other tasks that also require ordering; and three tests that do not require ordering. All non-Nback tasks were completed several days separate from the N-back by a mean interval (SD) of 5.14 (8.03). Our three hypotheses were that TGC during the N-back task would be associated with performance on N-Back and other cognitive tasks that also require ordering, but not with performance on cognitive tasks that do not require ordering; and that these relationships will be independent of clinical diagnosis. Multivariate linear regression results show that TGC was associated with performance on the ordering tasks but not the non-ordering tasks. In addition, there was no interaction between TGC and diagnosis. Our study is the first to demonstrate that TGC is a neurophysiologic measure of ordering information across several cognitive tasks that require ordering, and this TGC-ordering relationship is stable over time even when several days separate the measurement of TGC and the performance of the ordering tasks. Our results also show that this relationship is independent of clinical diagnosis, supporting the brain-behavior nature of this relationship. These results highlight the importance of TGC in ordering-based cognition, and suggest that TGC could be a valid target for interventions that aim to enhance this function across cognitive tasks and clinical conditions.
\end{abstract}

Neuropsychopharmacology (2020) 45:2038-2047; https://doi.org/10.1038/s41386-020-0759-z

\section{INTRODUCTION}

The ability to order information is a critical component in countless day-to-day activities. We code and maintain the order of information on a regular basis in order to accomplish simple tasks. For example, when following a recipe, it is not enough to know the individual ingredients, you must also know in what order the ingredients must be added. Further, individuals have a preference to remember information in a serial order even when the task demands do not require it $[1,2]$.

Ordering information is a key process that underlies several cognitive functions, such as working memory, executive functioning, episodic memory, and spatial navigation. During a working memory task, it is necessary to not only code the information to be remembered, but also code the sequential order of how the information is manipulated, e.g., during a mental arithmetic task.
Ordering information is also integral to episodic memory as what constitute an episodic memory is not only the events that occurred but also the sequence in which they occurred [3]. Coding for order is also essential in retrieving spatial information, for example, when following directions, you must remember not only how many left and right turns there are, but also the sequential order of the turns. Animal studies have demonstrated the importance of coding and maintaining serial order information during water maze tasks in rats [4, 5]. Finally, ordering of information is critical in several executive functioning tasks, e.g., planning, decision-making, and initiating goal-directed behavior $[6,7]$.

Neurophysiologically, brain oscillations and their relationships index ordering of information $[8,9]$. In particular, the modulation of oscillations at a certain frequency by oscillations at another

\footnotetext{
${ }^{1}$ Campbell Family Mental Health Research Institute, Centre for Addiction and Mental Health, Toronto, ON, Canada; ${ }^{2}$ Adult Neurodevelopment and Geriatric Psychiatry Division,

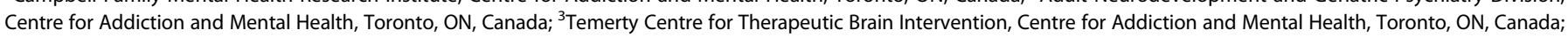

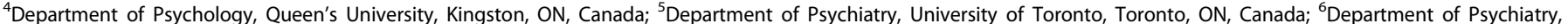

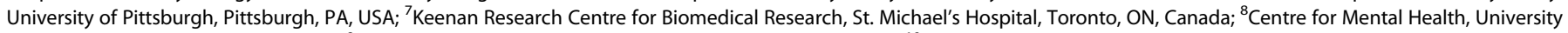

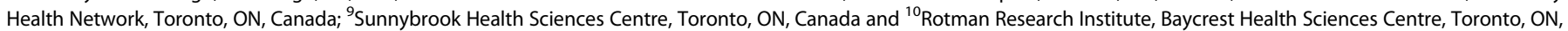
Canada

Correspondence: Tarek K. Rajji (Tarek.Rajji@camh.ca)
}

Received: 2 March 2020 Revised: 11 June 2020 Accepted: 2 July 2020

Published online: 18 July 2020 
A

$\stackrel{\mathrm{L}+\mathrm{A}+\mathrm{K}+\mathrm{A}+\mathrm{M}}{\longrightarrow}$

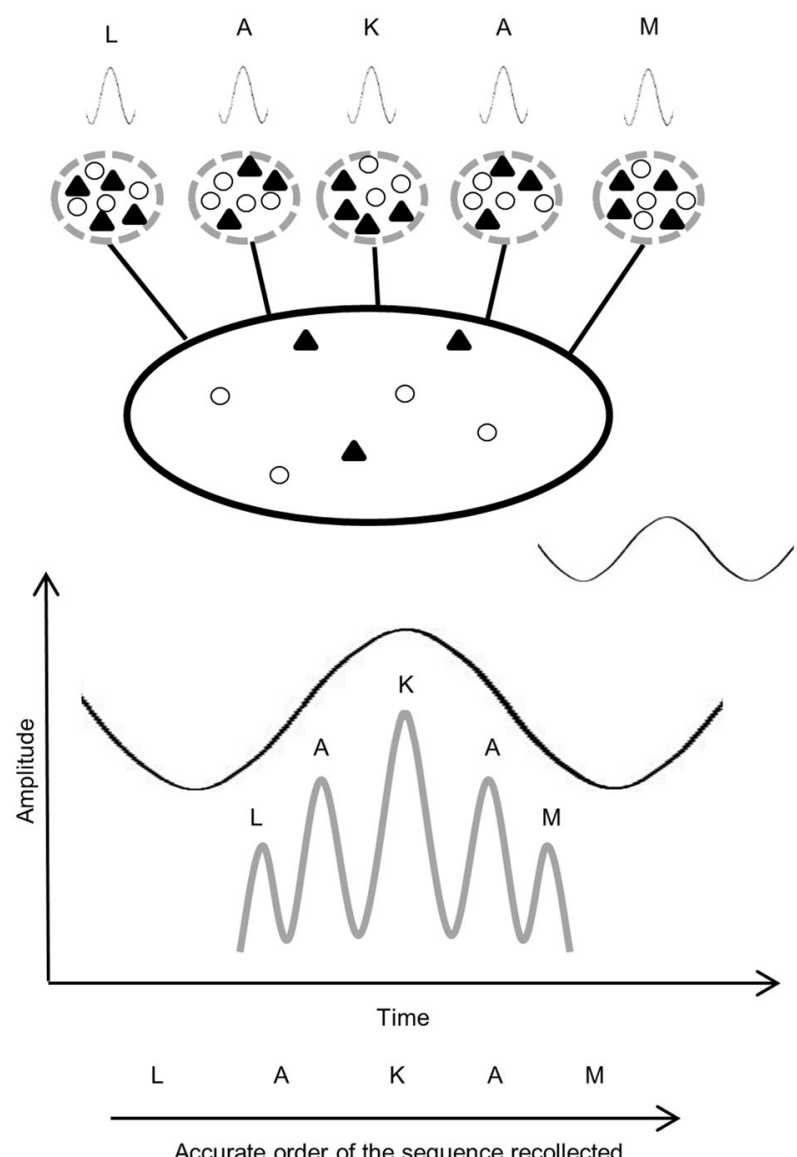

B

$\stackrel{\mathrm{L}+\mathrm{A}+\mathrm{K}+\mathrm{A}+\mathrm{M}}{\longrightarrow}$

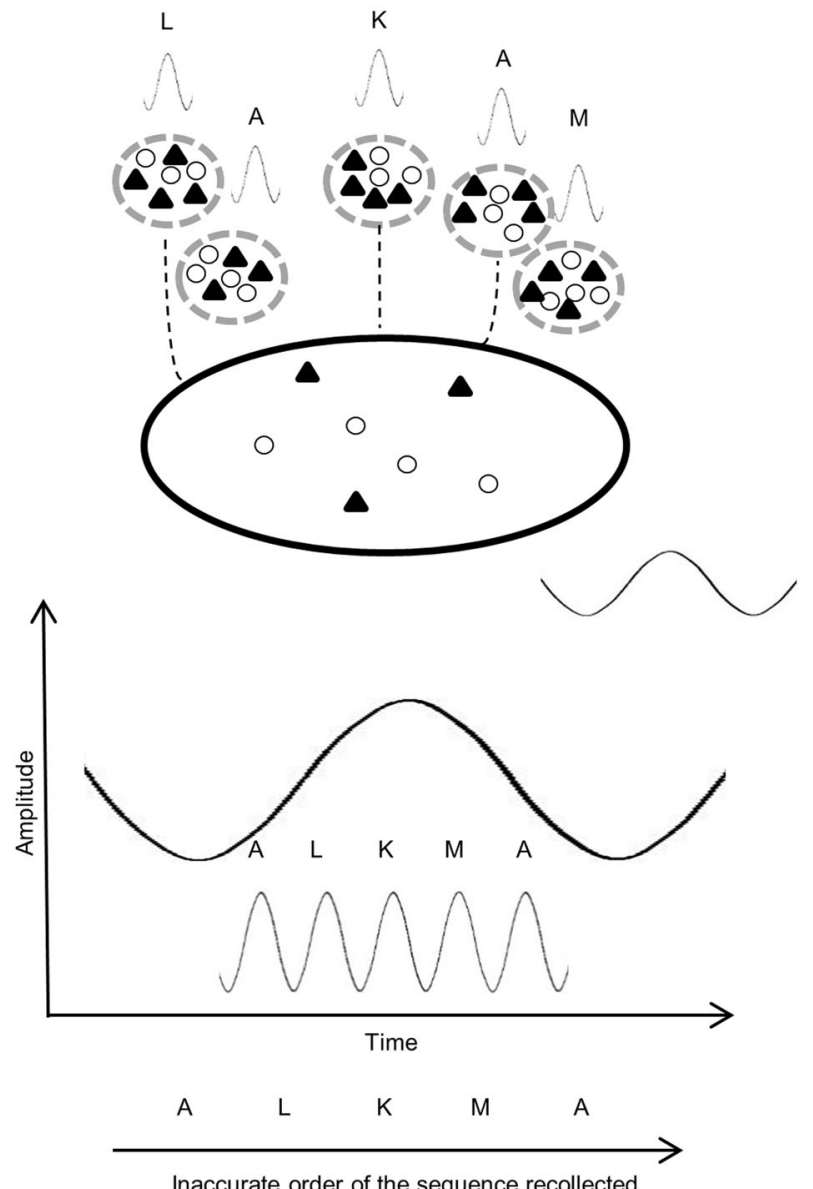

Fig. 1 Model of theta-gamma coupling during the $\mathbf{N}$-back task. a High theta-gamma coupling scenario: letters are presented in a sequential order and to answer correctly to a target trial (e.g., that the second A letter matches two letters back on a 2-back task), the participant needs to remember the order of this sequence correctly. In response to the presentation of these letters, each individual letter (one item of information) is represented by a unique pattern of activation of a neuronal assembly (small ellipse) of pyramidal and interneurons (black triangles and white circles). This activation results in a unique gamma oscillation that represents this letter. A different letter is then represented by a different pattern of activation of a different set of neurons, resulting in a different gamma oscillation. These different neuronal assemblies are intertwined with a common and larger neuronal assembly (large ellipse) the activation of which results in theta oscillations. This common and larger neuronal assembly, through its connections with the individual items neuronal assemblies, sets the order for sequential activation of the latter and thus, codes for the order in which the letters were presented during the N-back task. This larger assembly driven sequential activation of the smaller assemblies representing the letters results in coupling the unique gamma oscillations to specific phases of the theta oscillations, which, in turn, results in modulating gamma oscillations amplitudes by theta oscillation phase. The behavioral outcome of these intertwined activations of small and larger neuronal assemblies, and the corresponding coupling of gamma oscillations to theta oscillations, results in accurate recollection of the letters in their correct sequence. b Low theta-gamma coupling scenario: the neuronal assemblies representing the individual letters are not strongly intertwined with a common and larger assembly. Thus, their individual activations are not precisely ordered in a sequence that represents the sequence in which the letters were presented. In turn, the corresponding gamma oscillations are not coupled to an underlying oscillation generated by a network oscillating in activity at a slower frequency. i.e., theta. And consequently, the amplitudes of the gamma oscillations are not modulated by the phase of these theta oscillation, and the theta-gamma coupling index is low.

frequency, i.e., cross-frequency coupling, has been associated with ordering of information [10]. Theta-gamma coupling (TGC) is a form of cross-frequency coupling whereby high-frequency gamma $(30-50 \mathrm{~Hz})$ oscillations are modulated by low-frequency theta $(4-8 \mathrm{~Hz})$ oscillations [8]. Animal studies demonstrate that TGC represents the order of information, whereby low-frequency theta oscillations represent the time interval over which items are presented, and high-frequency gamma oscillations represent the individuals items of information over that time interval (see Fig. 1) [8, 11-13]. In humans, the relationship between TGC and ordering of information has also been established using working memory tasks and electroencephalography (EEG) during working memory task performance $[9,14]$. In one of the largest studies to date evaluating the relationship between TGC and ordering of information, we previously reported that TGC recorded from frontal electrodes during the performance of the N-back task is specifically high during the $\mathrm{N}$-back trials that require ordering of information in healthy adults [9]. Using a similar experimental setup, we also demonstrated that TGC predicts performance on the N-back in patients with Alzheimer's dementia (AD) and mild cognitive impairment (MCl) [14].

While the experimental setup of measuring TGC during performance of a working memory task clearly demonstrates an association between TGC and the ability to order information 
during a task, it is not clear whether using this approach to index TGC generalizes to other cognitive tasks that require ordering and that are not performed during TGC measurement. Such knowledge will be critical in validating TGC as a potential target for interventions to enhance cognitive performance beyond the task during which it is measured.

To address the above question, we took advantage of a wellcharacterized and relatively large sample of older individuals who are participating in a clinical trial to prevent $A D$ (NCT02386670) and who, at baseline, were tested using the Nback-EEG setup and a comprehensive neuropsychological battery. Each of these participants belonged to one of four diagnostic groups: $\mathrm{MCl}, \mathrm{MCl}$ with history of a major depressive disorder $(\mathrm{MCl}+\mathrm{MDD})$, history of MDD (MDD), or healthy control $(\mathrm{HC})$. Our primary aim was to examine the validity of TGC in measuring ordering of information by assessing its relationship with not only performance on the 2-back during which it was measured but also performance on other ordering tasks that were not administered during TGC measurement. To address this aim, we hypothesized that TGC during the N-back task (i.e., 2-back) will be associated with performance on 2-back and other cognitive tasks that also require ordering (Hypothesis $1(\mathrm{H} 1)$ ) but not with performance on cognitive tasks that do not require ordering $(\mathrm{H} 2)$. Our secondary aim was to examine whether the above the relationships hold true independent of diagnosis. To address this aim, we tested the hypothesis $(\mathrm{H} 3)$ of whether there is an interaction between diagnosis and TGC in their relationships to performances on cognitive tasks that do or do not require ordering.

\section{MATERIALS AND METHODS}

\section{Participants}

Participants were recruited as part of an $A D$ prevention trial (Prevention of AD with Cognitive Remediation plus Transcranial Direct Current Stimulation in Mild Cognitive Impairment and Depression (PACt-MD); NCT02386670) across five academic hospitals in Toronto, Canada. The full clinical trial design and rationale are published elsewhere [15]. All participants provided written informed consent as approved by the local Research Ethics Board and Clinical Trials Ontario. Participants were recruited using physician referrals, advertisements posted in local newspapers, magazines, hospitals and transit, and presentations given to community centers, retirement homes, and libraries.

Participants in the trial were recruited into one of three groups: $\mathrm{MCl}, \mathrm{MDD}$, and $\mathrm{HC}$. Participants in the MDD group were then classified (see details below) into MDD or $\mathrm{MCl}+\mathrm{MDD}$ resulting in four groups in total. Eligibility criteria for all participants were: (1) no lifetime Diagnostic and Statistical Manual of Mental Disorders Fifth Edition (DSM 5) [16] diagnosis of schizophrenia, bipolar disorder, or obsessive compulsive disorder; (2) no significant neurological conditions or unstable medical illnesses; (3) no DSM 5 diagnosis of alcohol or other substance use disorder within the past 12 months; (4) no use of a cognitive enhancers in the 6 weeks prior to entering the study; and (5) Montgomery-Asberg Depression Rating Scale [17] (MADRS) score of 10 or below.

Specific eligibility criteria for the $\mathrm{MCl}$ group were: (1) Age $\geq 60$; and (2) DSM 5 diagnosis of Minor Neurocognitive Disorder.

Specific eligibility criteria for the MDD group (with or without $\mathrm{MCl}$ ) were: (1) Age $\geq 65$; (2) having met DSM 5 criteria for one or more major depressive episode either: (a) occurring 2 months to 5 years from the screening date, or (b) occurring more than 5 years from the screening date with at least one of these episodes having been medically attended to (e.g., required an antidepressant or a hospitalization)—note in both scenarios the participant would not have met criteria for a current major depressive episode; and (3) no electroconvulsive therapy within 6 months of baseline neuropsychological testing.
Specific eligibility criteria for the HC group were: (1) Age $\geq 60$; (2) no DSM 5 diagnosis of Minor or Major Neurocognitive Disorder; and (3) no neuropsychological testing done in the 12 months prior to baseline assessment.

After meeting eligibility criteria and enrolling in the trial, each participant was discussed at a consensus conference that included the clinical psychiatrist of the participant, two principal investigators, the study neuropsychologist, and the research staff who administered the clinical, functional, and neuropsychological assessments. At the consensus conference, participants were classified into one of the following final four groups: (1) MCl only; (2) MDD with $\mathrm{MCl}(\mathrm{MCl}+\mathrm{MDD})$; (3) MDD without $\mathrm{MCl}(\mathrm{MDD})$; or (4) HC.

A total of $486 \mathrm{MCl}$ or MDD and $120 \mathrm{HC}$ participants consented to the parent study. The EEG study was an optional portion of the parent study, and a total of $384 \mathrm{MCl}$ or MDD and $78 \mathrm{HC}$ participants consented to the EEG study. These smaller numbers are because the EEG portion of the study did not start until after the parent study was launched, and because some participants declined to consent to this optional component of the parent study. Of those who consented to and completed the EEG at baseline, $264 \mathrm{MCl}$ or MDD and $47 \mathrm{HC}$ participants were included in the final analyses (see consort chart, Figs. 2 and 3).

\section{Assessments}

Clinical assessments. Diagnoses were confirmed using the Structured Clinical Interview for the DSM 5 (SCID-5) [18]. Participants also completed the Mini-Mental State Examination (MMSE) [19] and the Montreal Cognitive Assessment (MoCA) [20] to assess cognitive impairment clinically.

The Wide Range Achievement Test (WRAT 4)—Word Reading subtest was administered to estimate participants' premorbid IQ.

Neuropsychological assessments. All participants completed a battery of neuropsychological tasks. Neuropsychological assessments for all participants were completed by trained psychometrists at two study sites, with the exception of the N-Back EEG. The psychometrists at both sites were trained and certified by a PhD neuropsychologist (CRB). Assessments are video recorded, and reviewed periodically to ensure inter-rater reliability across the two sites $(\kappa=0.91)$. N-back EEGs for all participants were completed at one study site. A subset of tests were selected a priori from the overall battery to test our hypotheses. To assess the relationships between TGC and performance on cognitive tasks that require ordering of information, we selected the Paced Auditory Serial Addition Test (PASAT) [21] and the Trail Making Test A and B (TMT A and TMT B) [22].

The PASAT is a neuropsychological test that assesses attention, working memory, and speed of information processing, where a series of single-digit numbers are presented aurally and the participant is asked to sum the two most recent digits. Participants were administered two versions of the PASAT: one where participants have $2.4 \mathrm{~s}$ to give their response, and one where they have $1.6 \mathrm{~s}$ to respond. For this analysis, we used responses from the $2.4 \mathrm{~s}$ version, as the $1.6 \mathrm{~s}$ version is more difficult and participants had difficulty completing it, leading to more missing data. To perform correctly on the PASAT, participants need to maintain the order of the numbers presented and update the order with the new digits. The PASAT scores used for these analyses include some imputed values for individuals who refused to start or complete the task due to cognitive impairment. In these cases, the lowest observed value within each group (cases and controls) was assigned.

The TMT consists of two conditions: TMT A and TMT B. In TMT A, participants are instructed to draw lines sequentially connecting 25 encircled numbers distributed on a sheet of paper. In TMT B, participants must draw lines connecting both numbers and letters in alternating order (e.g., 1, A, 2, B, 3, C, etc.). Accurate performance 


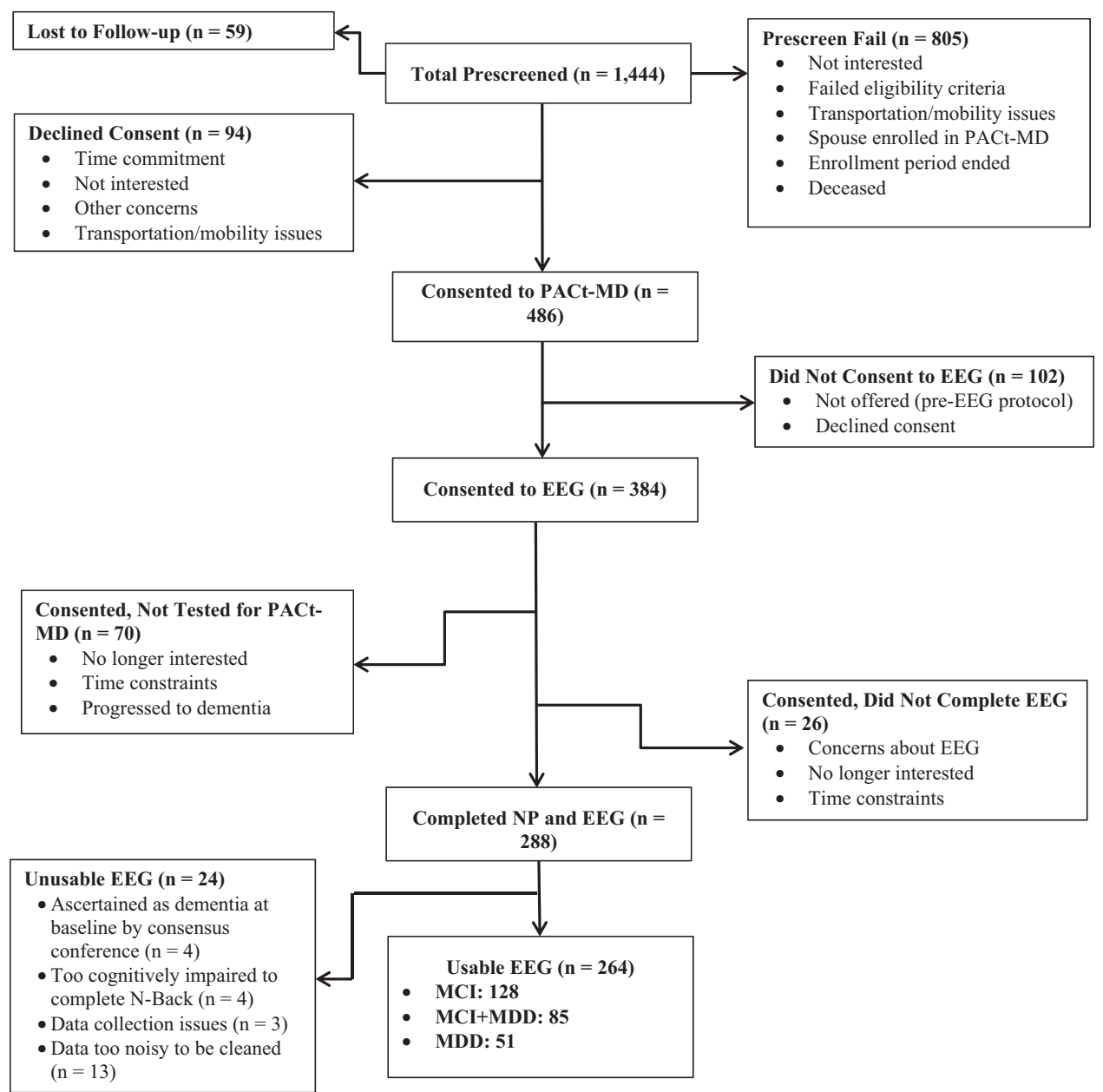

Fig. 2 Consort chart showing mild cognitive impairment (MCI), major depressive disorder (MDD) and MCI + MDD participants flow. PACtMD Prevention of Alzheimer's Dementia with Cognitive Remediation plus Transcranial Direct Current Stimulation in Mild Cognitive Impairment and Depression, EEG electroencephalography, NP neuropsychological testing.

on TMT requires participants to maintain the order of both the number and letter sequences. To assess executive function using TMT while controlling for information processing speed, we calculated TMT B/TMT $A$ ratio by dividing time per correct connection for TMT B by time per correct connection for TMT A [23].

In contrast to the above measures, we also selected neuropsychological tests that do not rely on the ability to code and maintain order of information to serve as control tests. These tests were the Boston Naming Test (BNT) [24], the Digit Symbol Coding test (DSC) [25], and the Judgment of Line Orientation (JLO) [26].

The BNT assesses verbal naming ability. Participants are shown pictures of common objects and are asked to name them. A split odd/even version BNT was used in this study [27], in which after the start point (item 30), participants were shown every other image, with the baseline version containing the odd-numbered items and received two points for a correct response. Items prior to the reverse rule were not split and received one point. Scores range from 0 to 60 , with higher scores indicating better performance on the test. The DSC test assesses information processing speed. Participants are shown a legend with numbers $1-9$, and below each number is a unique symbol that corresponds to that number. Below the legend, there are 135 boxes with numbers but no symbols. The participant has $120 \mathrm{~s}$ to fill in as many symbols as they can, with scores ranging from 0 to 135 . The JLO test assesses visuospatial processing and judgment. Participants are shown a set of line segments of varying spatial orientation and asked to match the lines to a set of longer lines on a response card. Scores range from 0 to 15 , with higher scores indicating more accurate performance. These three neuropsychological tests were chosen as control tasks because correct performance does not require ordering information.

\section{The $\mathrm{N}$-back task}

The N-back task is a continuous working memory task in which participants are required to determine if the stimulus presented on the screen is the same as, or different from the stimulus presented $\mathrm{N}$ trials back. The full experimental setup has been published elsewhere $[9,14,28]$. Similar to our previous publications using this sample of participants [14] we chose the 2-back condition as the primary condition for this analysis as it indexes working memory better than 0 or 1 -back condition and is not too hard, allowing it to generate more meaningful performance than the 3-back condition.

During the N-back task, trials where the stimulus presented matches the stimulus presented $\mathrm{N}$ trials back are labeled as target trials. Trials where the stimulus presented does not match the stimulus presented $\mathrm{N}$ trials back are labeled as nontarget trials.

EEG data recording and processing

EEG was recorded as participants completed the $\mathrm{N}$-back task using a 64-channel Synamps 2 EEG system and the 10-20 montage system. Electrodes were referenced to an electrode posterior to $\mathrm{CZ}$. EEG signals were recorded using DC and a low pass filter of $100 \mathrm{~Hz}$ at 20$\mathrm{kHz}$ sampling rate. EEG data processing occurred offline using 


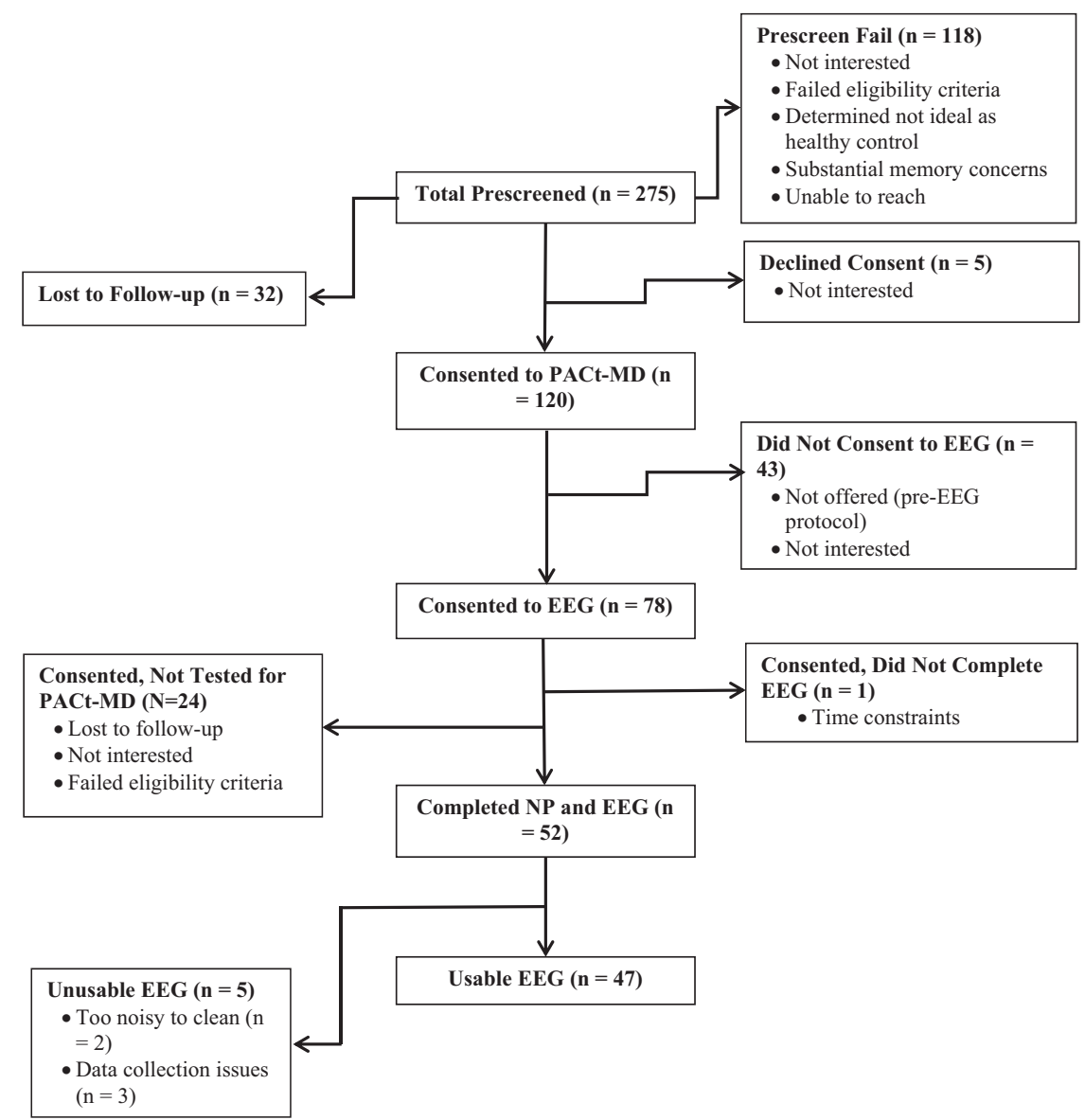

Fig. 3 Consort chart showing healthy control (HC) participant flow. PACt-MD Prevention of Alzheimer's Dementia with Cognitive Remediation plus Transcranial Direct Current Stimulation in Mild Cognitive Impairment and Depression, EEG electroencephalography, NP neuropsychological testing.

MATLAB (The MathWorks, Inc.) and EEGLAB toolbox. Data processing procedures have been published in full elsewhere $[9,14]$.

Theta-gamma coupling

The procedure for calculating the modulation index-the measure of TGC - has been described in detail elsewhere $[9,14,29]$. MI for each electrode was calculated, followed by an average of $\mathrm{MI}$ across the right and left frontal electrodes (F7/8, F5/F6, F3/4, F1/2, and Fz). Following previously published methods, Ml for all target trials was analyzed as a weighted average based on the number of correct and incorrect responses [14].

Using a similar N-back-EEG setup, our previous studies demonstrated that TGC in the frontal region during the $\mathrm{N}$-back task is highest on target trials, when recalling the exact order of information is necessary for correct performance. In contrast, TGC was lowest on nontarget trials when recalling the specific order of information is neither necessary nor beneficial to performance $[9,14]$. Therefore, we used TGC during the target trials during which more ordering and, in turn, more pronounced TGC, occurs than during the nontarget ones.

Theta and gamma powers

In order to determine to what extent TGC predicts ordering-based cognition independently from theta or gamma power, theta and gamma powers were also calculated. Once the signal was filtered into the appropriate bands, the squared amplitude of Hilbert transformed signal was used to calculate the power spectrum. A group average of spectral power for all frontal electrodes, weighted by response type, for target trials was then calculated.
Statistical analyses

All data were analyzed using the Statistical Program for Social Sciences (SPSS) version 24.0 (SPSS Inc., Chicago, IL, USA). All neuropsychological variables were converted into $T$ scores using the mean and standard deviation of all groups combined. Data were visually inspected for normal distribution, and were checked statistically with the Shapiro-Wilk test. All non-normal data were transformed using a natural log transformation in order to approximate a normal distribution. The following data were transformed: weighted MI values; theta and gamma powers; and the TMT B/TMT A ratio. We also conducted Pearson correlations between performance on all neuropsychological variables.

Chi-square and one-way analysis of variance (ANOVA) were run to determine if there were any significant differences between the groups on any of the demographic, clinical, neuropsychological, or neurophysiologic variables. Significant ANOVAs were then followed up with post-hoc tests to determine where the significant differences lie.

To address $\mathrm{H} 1$ and $\mathrm{H} 2$, we conducted a multivariate linear regression model with six dependent variables: 2-back Dprime, PASAT, and TMT B/TMT A to address $\mathrm{H} 1$; and JLO, DSC, and BNT to address $\mathrm{H} 2$. The model also included seven independent variables: $\mathrm{Ml}$, gamma and theta power, age, education WRAT 4 score, and diagnosis. Age, education, and premorbid IQ are known to have an impact on performance on neuropsychological tests, thus they were included in the model as independent variables. Theta and gamma powers were included to determine to what extent TGC is associated with ordering-based cognition independently of theta and gamma powers. 
Table 1. Demographic, Clinical, Cognitive, and Neurophysiologic Data.

\begin{tabular}{|c|c|c|c|c|c|c|c|}
\hline & $\begin{array}{l}\mathrm{MCl} \\
(n=128)\end{array}$ & $\begin{array}{l}\text { MDD } \\
(n=51)\end{array}$ & $\begin{array}{l}\mathrm{MCl}+\mathrm{MDD} \\
(n=85)\end{array}$ & $\begin{array}{l}\mathrm{HC} \\
(n=47)\end{array}$ & $F$ or $X^{2}(\mathrm{df})$ & $p$ value & Post-hoc comparisons \\
\hline Age & $71.96(7.23)$ & 69.27 (3.99) & $70.85(4.68)$ & $69.70(5.66)$ & $3.30(3,307)$ & $0.02^{\mathrm{a}}$ & $\mathrm{MCl}>\mathrm{MDD}$ \\
\hline \multicolumn{5}{|l|}{ Highest level of education } & $13.20(18)$ & 0.78 & - \\
\hline Less than high school & 7 & 3 & 4 & 1 & & & \\
\hline High school graduate & 13 & 3 & 9 & 2 & & & \\
\hline Partial University & 10 & 8 & 9 & 2 & & & \\
\hline University degree & 65 & 24 & 43 & 28 & & & \\
\hline Graduate degree & 33 & 14 & 20 & 13 & & & \\
\hline Gender (M:F) & $51: 78$ & $15: 37$ & $32: 53$ & $15: 32$ & $2.29(3)$ & 0.51 & - \\
\hline MMSE & $27.90(1.62)$ & $29.00(1.22)$ & $28.06(1.55)$ & $29.04(1.16)$ & $11.68(3,305)$ & $<0.001^{\mathrm{b}}$ & $\mathrm{HC}, \mathrm{MDD}>\mathrm{MCl}, \mathrm{MCl}+\mathrm{MDD}$ \\
\hline MoCA & $23.77(2.45)$ & $27.60(1.73)$ & $24.76(2.64)$ & $27.77(1.29)$ & $57.40(3,307)$ & $<0.001^{\mathrm{b}}$ & $\begin{array}{l}\mathrm{HC}, \mathrm{MDD}>\mathrm{MCl}, \mathrm{MCl}+\mathrm{MDD} \\
\mathrm{MCl}+\mathrm{MDD}>\mathrm{MCl}\end{array}$ \\
\hline WRAT 4 Score & $62.98(4.57)$ & $65.49(3.67)$ & $64.00(4.73)$ & $65.72(3.48)$ & $6.83(3,307)$ & $<0.001^{\mathrm{b}}$ & $\mathrm{HC}, \mathrm{MDD}>\mathrm{MCl}, \mathrm{MCl}+\mathrm{MDD}$ \\
\hline 2-back DPrime $T$ score & $48.57(9.32)$ & $52.15(10.18)$ & 47.06 (9.79) & $57.55(7.54)$ & $14.66(3,305)$ & $<0.001^{\mathrm{b}}$ & $\begin{array}{l}\mathrm{HC}>\mathrm{MCl}, \mathrm{MDD}, \mathrm{MCl}+\mathrm{MDD} \\
\mathrm{MDD}>\mathrm{MCl}+\mathrm{MDD}\end{array}$ \\
\hline PASAT $T$ score & $48.00(9.75)$ & $51.80(9.10)$ & $48.14(10.42)$ & $56.30(7.76)$ & $10.21(3,294)$ & $<0.001^{\mathrm{b}}$ & $\mathrm{HC}>\mathrm{MCl}, \mathrm{MCl}+\mathrm{MDD}$ \\
\hline TMT B/TMT A $T$ score & $48.93(3.80)$ & $47.87(3.64)$ & $49.65(4.51)$ & $47.56(2.84)$ & $3.90(3,293)$ & $0.009^{\mathrm{b}}$ & $\mathrm{HC}>\mathrm{MCl}+\mathrm{MDD}$ \\
\hline BNT $T$ score & $48.40(9.53)$ & $53.80(6.89)$ & $50.98(7.50)$ & $53.53(6.65)$ & $7.59(3,299)$ & $<0.001^{\mathrm{b}}$ & $\mathrm{HC}, \mathrm{MDD}>\mathrm{MCl}$ \\
\hline JLO-Q T score & $49.08(10.66)$ & $52.76(8.21)$ & $47.66(9.85)$ & $53.43(9.05)$ & $5.17(3,305)$ & $0.002^{\mathrm{b}}$ & $\mathrm{HC}>\mathrm{MCl} ; \mathrm{MDD}>\mathrm{MCl}+\mathrm{MDD}$ \\
\hline DSC $T$ score & $48.44(9.62)$ & $54.46(8.64)$ & $46.09(9.44)$ & $56.46(8.33)$ & $17.87(3,305)$ & $<0.001^{\mathrm{b}}$ & $\mathrm{HC}, \mathrm{MDD}>\mathrm{MCl}$ \\
\hline 2-Back MI & $0.0033(0.005)$ & $0.0035(0.003)$ & $0.0033(0.003)$ & $0.0047(0.004)$ & $4.17(3,305)$ & $0.006^{\mathrm{b}}$ & $\mathrm{HC}>\mathrm{MCl}, \mathrm{MCl}+\mathrm{MDD}$ \\
\hline 2-Back gamma power & $6722.93(9847.9)$ & $5138.62(4126.1)$ & 6371.52 (7313.7) & $4472.77(4292.3)$ & $1.51(3,306)$ & 0.08 & - \\
\hline 2-Back theta power & $16,304.24(14281.3)$ & $15,805.84(11888.9)$ & $19,338.40(24290.3)$ & $18,556.87(24026.7)$ & $0.37(3,306)$ & 0.69 & - \\
\hline \multicolumn{8}{|c|}{$\begin{array}{l}\text { Except for education and gender, values are listed as mean (SD). } \\
M C I \text { mild cognitive impairment, Mdd Major depressive disorder, } M C I+M D D \text { Mild cognitive impairment }+ \text { major depressive disorder, } H C \text { healthy control, MMSE } \\
\text { Mini-Mental State Examination, MoCA Montreal Cognitive Assessment, MI Modulation Index, WRAT } 4 \text { Wide Range Achievement Test } 4-\text { Word Reading subtest, } \\
\text { PASAT Paced Auditory Serial Addition Test, TMT B/TMT A Trail Making Test Part B time-per-connection ratio divided by Trail Making Test Part A time-per- } \\
\text { connection ratio, BNT Boston Naming Test, DSC digit symbol coding, JLO-Q Judgment of Line Orientation-Q, F one-way analysis of variance, } X^{2} \text { Chi-square test, } \\
d f \text { degrees of freedom. } \\
\text { andicates significance at } a \leq 0.05 \text {. } \\
{ }^{\mathrm{b}} \text { Indicates significance at } a \leq 0.01 \text {. }\end{array}$} \\
\hline
\end{tabular}

To determine whether the relationship between TGC and ordering exists independent of diagnosis $(\mathrm{H} 3)$, we ran another model, which included an interaction term between TGC and diagnosis as an independent variable.

\section{RESULTS}

Demographic, clinical, cognitive, and neurophysiological data and the results of the group comparisons are presented in Table 1. On the demographic and clinical measures, there were differences across diagnoses in age, MMSE, MoCA, and WRAT 4 scores. On the cognitive measures, there were differences in performance across diagnoses on all tasks. On the neurophysiological measures, there were differences across diagnoses in $\mathrm{Ml}$, but not theta or gamma power (see Table S1). Correlations between performance on the neuropsychological variables is presented in Table S2.

TGC and performance on ordering and non-ordering tasks The results of the multivariate linear regression are presented in Table 2. As hypothesized, the model shows an association between TGC and performance on the ordering tasks: 2-back Dprime $(B=4.36, p<0.001)$, PASAT $(B=2.35, p=0.005)$, and TMT $\mathrm{B} / \mathrm{TMT} \mathrm{A}$ ratio $(B=-0.02, p=0.03)$; and no association with performance on the non-ordering tasks: JLO $(B=0.76, p=0.37)$, DSC $(B=0.20, p=0.79)$, or BNT $(B=-0.24, p=0.74)$.

The model also shows age, education, and WRAT 4 contribute to the overall model. Age is associated with performance on all of the neuropsychological tests (2-back Dprime: $B=-0.42, p<$ 0.001 ; TMT B/TMT A: $B=0.002, p=0.003$; DSC: $B=-0.55, p<$
0.001 ; BNT: $B=-0.17, p=0.03)$, except for the JLO $(B=-0.15$, $p=0.12)$. Education is associated with performance on the PASAT $(B=1.49, p=0.01)$, TMT B/TMT A $(B=-0.01, p=0.03)$, and DSC $(B=1.39, p=0.01)$. WRAT 4 score is associated with performance on all neuropsychological tests (2-back Dprime: $B=0.46, p<0.001$; PASAT: $B=0.27, p=0.05$; TMT B/TMT A: $B=$ $-0.002, p=0.03$; JLO: $B=0.48, p<0.001$; DSC: $B=0.36, p=$ 0.006 ; BNT: $B=0.70, p<0.001)$.

TGC and diagnosis

The model shows that diagnosis contributes to the overall model (Pillai's Trace $=0.18, F(18,792)=2.81, p<0.001$ ), showing that performance on the neuropsychological tests differs by diagnosis. The diagnosis $\times \mathrm{Ml}$ interaction term, however, does not contribute to the overall model (Pillai's Trace $=0.05, F(18,793)=0.77, p=$ 0.74; see Table 2).

\section{DISCUSSION}

In this study, we examined the relationship between TGC as measured during the 2-back and its relationship to ordering of information, not only with performance on the task during which it is measured, but also performance as measured by other neuropsychological assessments. Our results are consistent with our earlier work, which showed that there is a significant association between TGC measured during the 2-back and performance on the 2-back [14]. Further, our results show that TGC on the 2-back, where ordering is necessary for correct performance, is associated with performance on two cognitive tasks which also require ordering of information. In addition, we 
Table 2. Multivariate linear regression results.

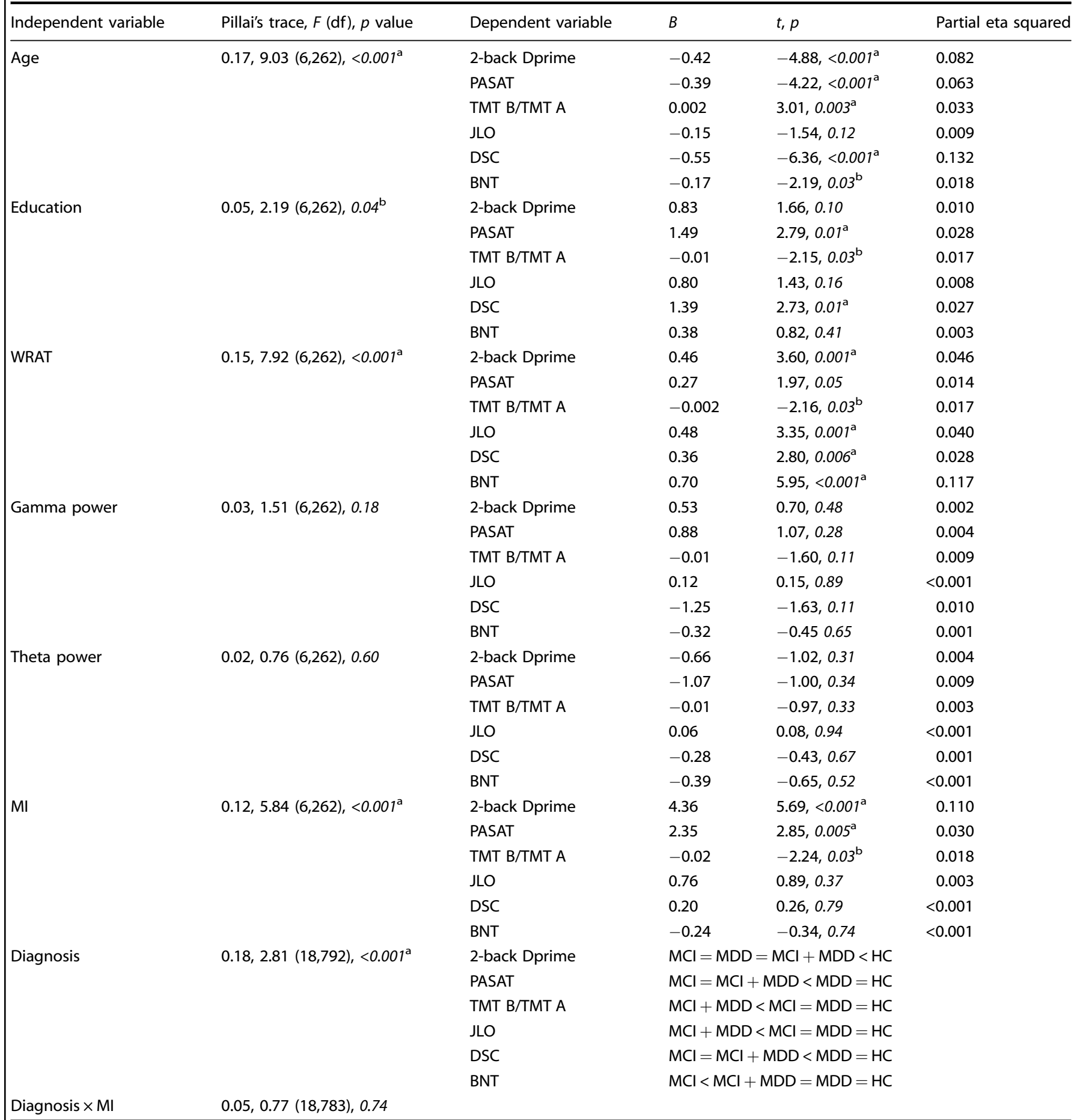

$F$ one-way ANOVA, $d f$ degrees of freedom, $B$ Uunstandardized beta, $t$ independent-samples $t$ test, $p$ p-value, WRAT 4 Wide Range Achievement Test 4-Word Reading subtest, MI Modulation index, PASAT Paced Auditory Serial Addition Test, TMT B/TMT A Trail Making Test Part B time-per-connection ratio divided by Trail Making Test Part A time-per-connection ratio, BNT Boston Naming Test, DSC digit symbol coding, JLO-Q Judgment of Line Orientation-Q.

andicates significance at $a \leq 0.01$.

b Indicates significance at $a<0.05$.

found that TGC was not associated with performance on cognitive tasks that do not require ordering of information. Our findings support the role of TGC as a neurophysiologic mechanism that underlies the cognitive process of ordering of information across different cognitive tasks. In addition, our results show there is no significant interaction between diagnosis and TGC, which suggest the relationship between TGC and ordering of information is independent of diagnosis. Taken together, our results suggest that the relationship between TGC and ordering of information is not only independent of task but also independent of clinical 
condition and that it most likely indexes a specific brain-behavior relationship.

Our findings extend our previous studies on TGC and ordering of information in humans. In one study, we examined the role of TGC in ordering information during the $\mathrm{N}$-back task in a group of healthy individuals. Results showed that TGC was highest during target trials and lowest during nontarget trials, and that TGC significantly predicted performance on target trials [9]. In a second study, we examined the relationship between TGC and performance on the $\mathrm{N}$ back task in healthy individuals vs. $\mathrm{MCl}$ and $\mathrm{AD}$ participants. Results showed that TGC on target trials was a strong predictor of performance on the 2-back in these populations and that it differentiated healthy from disease states [14]. These two studies examined TGC during the N-back task and performance on the $\mathrm{N}$ back task. The results of this study further extend the validity of TGC as a neurophysiologic measure of ordering of information by demonstrating that TGC as measured during the 2-back task is associated with performance on other cognitive tasks that require ordering and that were completed several days from the 2-back.

Preclinical and computational studies of TGC suggest that gamma oscillations are generated by subsets of neuronal assemblies, each representing an individual item of information. The order of activation of these neuronal assemblies is supported by a longer-range organizational network which activity is captured by theta oscillations. As such, the order is coded by theta phase-modulation of gamma oscillations amplitude [11, 30]. In our study, we are able to capture this neurophysiologic phenomenon using frontal EEG electrodes and this frontal TGC is associated with performance on a working memory test, the PASAT. This association is consistent with frontal lobes activation using fMRI during PASAT performance [31, 32]. Similarly, frontal TGC association with performance on TMT is consistent with frontal lobes activation during $\mathrm{fMRI}$ and TMT performance [33, 34].

This study has some limitations. One limitation is the crosssectional design of the study. Although we see a relationship between TGC and ordering of information cross-sectionally, longitudinal studies are needed to determine if this relationship is stable over time, and to assess whether enhancing TGC could lead to improvement in these ordering-based cognitive tasks. Another limitation is the heterogeneous nature of our sample. For example, our $\mathrm{MCl}$ sample included both amnestic and nonamnestic MCl. Similarly, the MDD group was quite heterogeneous, with varying clinical differences, such as age of onset and number of episodes. Future studies could examine these relationships in a more biologically defined sample. However, this heterogeneity allowed us to address the question of whether TGC-ordering relationship is independent of clinical condition, and our results support this hypothesis. While the two ordering tasks chosen for this study (i.e., PASAT and TMT) assess cognitive domains other than working memory, they both also assess working memory. Thus, it is possible that the associations we found between TGC and performance on the PASAT and TMT are reflecting associations between TGC and working memory using two working memory tasks other than the N-back. Still, the novelty of this finding would be that TGC is associated with working memory beyond the task during which it is measured. Future research should include control tasks that assess working memory but do not have an ordering component, such as a logical memory test, or an ordering task that does not have a working memory component to help determine if TGC is associated with working memory more generally, or ordering. Last, the absence of cognitive tasks which require participants to generate ordered stimuli, such as Letter Number Span, as opposed to maintaining the order of stimuli, are missing in this study. The generation of ordered stimuli vs. the maintenance of ordered stimuli are similar yet distinct processes. Future research should include tasks that require the generation of ordered stimuli to determine if there is a relationship between TGC and generation, in addition to the relationship between TGC and the maintenance and recollection of ordered stimuli.

In conclusion, our study is the first to demonstrate that TGC is a neurophysiologic measure of ordering information, not only in the task during which it was measured, but across other cognitive tasks where ordering is required. The findings of the current study could be used to inform future interventions, by using TGC as a marker for intervention. If TGC is associated with ordering of information on different tasks, improving TGC through intervention could enhance ordering, and thus, improve cognitive performance on ordering tasks. Future research should examine the utility of using TGC as a potential marker for intervention in populations at high-risk for cognitive decline. Prior research demonstrates that TGC has been shown to respond to different neurostimulation interventions, such as paired associative stimulation [35], repetitive transcranial magnetic stimulation [36], and transcranial alternating current stimulation [37]. By improving TGC through interventions, such as brain stimulation, it may be possible to improve cognitive function associated with ordering, which could potentially slow cognitive decline.

\section{FUNDING AND DISCLOSURE}

This work has been made possible by Brain Canada through the Canada Brain Research Fund, with the financial support of Health Canada and the Chagnon Family; Canada Foundation for Innovation (\#25861, PI: TKR); Canadian Institutes of Health Research (\#244041, PI: TKR); the Ontario Ministry of Research and Innovation (\# ER14-10-004, PI: TKR); and the Joan and Clifford Hatch Foundation. DMB has received research support from the $\mathrm{CIHR}, \mathrm{NIH}$, Brain Canada and the Temerty Family Foundation through the CAMH Foundation and the Campbell Research Institute. He received research support and in-kind equipment support for an investigator-initiated study from Brainsway Ltd, and he is the principal site investigator for three sponsor-initiated studies for Brainsway Ltd. He received in-kind equipment support from Magventure for investigator-initiated research. He received medication supplies for an investigator-initiated trial from Indivior. He has participated in an advisory board for Janssen. ZJD has received research and equipment in-kind support for an investigator-initiated study through Brainsway Inc and Magventure Inc. His work was supported by the Ontario Mental Health Foundation (OMHF), the Canadian Institutes of Health Research (CIHR), the National Institutes of Mental Health (NIMH) and the Temerty Family and Grant Family and through the Centre for Addiction and Mental Health (CAMH) Foundation and the Campbell Institute. CEF receives grant funding from Hoffman LaRoche and Vielight Inc. AJF has received grant support from the U.S. National Institutes of Health, the Patient-Centered Outcomes Research Institute, the Canadian Institutes of Health Research, Brain Canada, the Ontario Brain Institute, and Alzheimer's Association. SK has received grant support from Brain Canada, $\mathrm{NIH}$, Brain and Behavior Foundation (NARSAD), BrightFocus Foundation, Weston Brain Institute, Canadian Centre for Ageing and Brain Health Innovation, CAMH foundation and University of Toronto, and in Kind equipment support from Soterix Medical Inc. BHM currently receives research support from Brain Canada, the Canadian Institutes of Health Research, the CAMH Foundation, the Patient-Centered Outcomes Research Institute (PCORI), the US National Institute of Health (NIH), Capital Solution Design LLC (software used in a study founded by CAMH Foundation), and HAPPYneuron (software used in a study founded by Brain Canada). He directly own stocks of General Electric $(<\$ 5000)$. Within the past 3 years, he has also received research support from Eli Lilly (medications for a $\mathrm{NIH}$-funded clinical trial) and Pfizer (medications for a NIH-funded clinical trial). TKR has received research support from Brain Canada, Brain and Behavior Research Foundation, BrightFocus Foundation, Canada Foundation for 
Innovation, Canada Research Chair, Canadian Institutes of Health Research, Centre for Aging and Brain Health Innovation, National Institutes of Health, Ontario Ministry of Health and Long-Term Care, Ontario Ministry of Research and Innovation, and the Weston Brain Institute. TKR also received in-kind equipment support for an investigator-initiated study from Magstim, and in-kind research accounts from Scientific Brain Training Pro. HB, MSG, CRB, RZ, $M A B, N H, L M, B G P$, and $A V$ report no competing interests.

\section{ACKNOWLEDGEMENTS}

This work has been made possible by Brain Canada through the Canada Brain Research Fund, with the financial support of Health Canada and the Chagnon Family; Canada Foundation for Innovation (\#25861, PI: TKR); Canadian Institutes of Health Research (\#244041, PI: TKR); the Ontario Ministry of Research and Innovation (\# ER1410-004, PI: TKR); and the Joan and Clifford Hatch Foundation. The authors would like to thank the entire PACt-MD Study Group: Benoit H. Mulsant, Tarek K. Rajji, Nathan Herrmann, Bruce G. Pollock, Lillian Lourenco, Daniel M. Blumberger, Christopher R. Bowie, Meryl Butters, Corinne Fischer, Alastair Flint, Damian Gallagher, Angela Golas, Ariel Graff, James L. Kennedy, Sanjeev Kumar, Linda Mah, Shima Ovaysikia, Mark. Rapoport, Kevin Thorpe, Nicolaas P.L.G. Verhoeff, Aristotle N. Voineskos.

\section{AUTHOR CONTRIBUTIONS}

$\mathrm{HB}$ analyzed the data and wrote the paper. HB and TKR reviewed the first draft. MSG, RZ, SK, and TKR developed the analysis methods. BHM, TKR, DMB, and CRB developed the design of the parent study. BHM, TKR, DMB, CEF, AF, LM, NH, SK, and CRB contributed to the recruitment and assessment of participants. ZJD, AV, MAB, BGP, and all other co-authors aided in interpretation of the results and editing of the paper. All authors reviewed and approved the final paper.

\section{ADDITIONAL INFORMATION}

Supplementary Information accompanies this paper at (https://doi.org/10.1038/ s41386-020-0759-z).

Publisher's note Springer Nature remains neutral with regard to jurisdictional claims in published maps and institutional affiliations.

\section{REFERENCES}

1. Ward G, Tan L, Grenfell-Essam R. Examining the relationship between free recall and immediate serial recall: the effects of list length and output order. J Exp Psychol Learn Mem Cogn. 2010;36:1207.

2. Grenfell-Essam R, Ward G. Examining the relationship between free recall and immediate serial recall: the role of list length, strategy use, and test expectancy. J Mem Lang. 2012;67:106-48.

3. Eichenbaum H. A cortical-hippocampal system for declarative memory. Nat Rev Neurosci. 2000;1:41.

4. Bott J-B, Muller M-A, Jackson J, Aubert J, Cassel J-C, Mathis C, et al. Spatial reference memory is associated with modulation of theta-gamma coupling in the dentate gyrus. Cereb Cortex. 2016;26:3744-53.

5. Hannesson D, Vacca G, Howland J, Phillips A. Medial prefrontal cortex is involved in spatial temporal order memory but not spatial recognition memory in tests relying on spontaneous exploration in rats. Behav Brain Res. 2004;153: 273-85.

6. Lashley KS. The problem of serial order in behavior. Oxford, UK: Bobbs-Merrill; 1951.

7. Hurlstone MJ, Hitch GJ, Baddeley AD. Memory for serial order across domains: an overview of the literature and directions for future research. Psychol Bull. 2014;140:339.

8. Tort AB, Komorowski RW, Manns JR, Kopell NJ, Eichenbaum H. Theta-gamma coupling increases during the learning of item-context associations. Proc Natl Acad Sci. 2009;106:20942-47.

9. Rajji TK, Zomorrodi R, Barr MS, Blumberger DM, Mulsant BH, Daskalakis ZJ. Ordering information in working memory and modulation of gamma by theta oscillation in humans. Cereb Cortex. 2017;27:1482-90.

10. Canolty RT, Knight RT. The functional role of cross-frequency coupling. Trends Cogn Sci. 2010;14:506-15.
11. Lisman JE, Jensen O. The theta-gamma neural code. Neuron. 2013;77:1002-16.

12. Shirvalkar PR, Rapp PR, Shapiro ML. Bidirectional changes to hippocampal theta-gamma comodulation predict memory for recent spatial episodes. Proc Natl Acad Sci. 2010;107:7054-59.

13. Jensen $\mathrm{O}$, Tesche $C D$. Frontal theta activity in humans increases with memory load in a working memory task. Eur J Neurosci. 2002;15:1395-99.

14. Goodman MS, Kumar S, Zomorrodi R, Ghazala Z, Cheam ASM, Barr MS, et al. Theta-gamma coupling and working memory in Alzheimer's dementia and mild cognitive impairment. Front Aging Neurosci. 2018;10:101.

15. Rajji TK, Bowie CR, Herrmann N, Pollock BG, Bikson M, Blumberger DM, et al. Design and Rationale of the PACt-MD randomized clinical trial: prevention of Alzheimer's dementia with cognitive remediation plus transcranial direct current stimulation in mild cognitive impairment and depression. J Alzheimer's Dis. 2020:1-29. In press.

16. Association AP. Diagnostic and statistical manual of mental disorders (DSM-5 $5^{\circ}$ ). American Psychiatric Association Publishing; 2013.

17. Montgomery SA, Åsberg M. A new depression scale designed to be sensitive to change. Br J Psychiatry. 1979;134:382-89.

18. First M, Williams J, Karg R, Spitzer R. Structured clinical interview for DSM-5research version (SCID-5 for DSM-5, research version; SCID-5-RV). Arlington, VA: American Psychiatric Association; 2015.

19. Folstein MF, Folstein SE, McHugh PR. "Mini-mental state": a practical method for grading the cognitive state of patients for the clinician. J Psychiatr Res. 1975;12:189-98.

20. Nasreddine ZS, Phillips NA, Bédirian V, Charbonneau S, Whitehead V, Collin I, et al. The Montreal Cognitive Assessment, MoCA: a brief screening tool for mild cognitive impairment. J Am Geriatrics Soc. 2005;53:695-99.

21. Gronwall D. Paced auditory serial-addition task: a measure of recovery from concussion. Percept Mot Skills. 1977;44:367-73.

22. Strauss $E$, Sherman EM, Spreen O. A compendium of neuropsychological tests: administration, norms, and commentary. American Chemical Society; Journal of clinical and experimental neuropsychology; 2006.

23. Arbuthnott K, Frank J. Trail making test, part B as a measure of executive control: validation using a set-switching paradigm. J Clin Exp Neuropsychol. 2000;22: 518-28.

24. Kaplan E, Goodglass H, Weintraub S. Boston naming test. Pro-Ed Inc; 2001.

25. Wechsler D. Wechsler Adult Intelligence Scale-Fourth Edition (WAIS-IV). San Antonio, TX: The Psychological Corporation; 2008.

26. Benton AL, Hamsher K, Varney NR, Spreen O. Judgment of line orientation. New York: Oxford University Press; 1983.

27. Fisher NJ, Tierney MC, Snow GW, Szalai JP. Odd/even short forms of the Boston naming test: preliminary geriatric norms. Clin Neuropsychol. 1999;13:359-64.

28. Barr MS, Farzan F, Rajji TK, Voineskos AN, Blumberger DM, Arenovich T, et al. Can repetitive magnetic stimulation improve cognition in schizophrenia? Pilot data from a randomized controlled trial. Biol Psychiatry. 2013;73:510-17.

29. Axmacher N, Henseler MM, Jensen O, Weinreich I, Elger CE, Fell J. Cross-frequency coupling supports multi-item working memory in the human hippocampus. Proc Natl Acad Sci. 2010;107:3228-33.

30. Rajji TK, Zomorrodi R, Barr MS, Blumberger DM, Mulsant BH, Daskalakis ZJ. Ordering information in working memory and modulation of gamma by theta oscillations in humans. Cereb Cortex. 2017;27:1482-90.

31. Cardinal KS, Wilson SM, Giesser BS, Drain AE, Sicotte NL. A longitudinal fMRI study of the paced auditory serial addition task. Mult Scler. 2008;14:465-71.

32. Audoin B, Ibarrola D, Duong MVA, Pelletier J, Confort-Gouny S, Malikova I, et al. Functional MRI study of PASAT in normal subjects. Magn Reson Mater Phys Biol Med. 2005;18:96-102.

33. Moll J, de Oliveira-Souza R, Moll FT, Bramati IE, Andreiuolo PA. The cerebral correlates of set-shifting-an fMRI study of the trail making test. Arquivos De Neuro-Psiquiatria. 2002;60:900-5.

34. Zakzanis KK, Mraz Z, Graham S. An fMRI study of the Trail Making Test. Neuropsychologia. 2005;43:1878-86.

35. Rajji TK, Sun Y, Zomorrodi-Moghaddam R, Farzan F, Blumberger DM, Mulsant $\mathrm{BH}$, et al. PAS-induced potentiation of cortical evoked activity in the dorsolateral prefrontal cortex. Neuropsychopharmacology. 2013;38: 2545-52.

36. Noda Y, Zomorrodi R, Saeki T, Rajji TK, Blumberger DM, Daskalakis ZJ, et al. Resting-state EEG gamma power and theta-gamma coupling enhancement following high-frequency left dorsolateral prefrontal rTMS in patients with depression. Clin Neurophysiol. 2017;128:424-32.

37. Reinhart RM, Nguyen JA. Working memory revived in older adults by synchronizing rhythmic brain circuits. Nat Neurosci. 2019;1:820-7. 
ON BEHALF OF THE PACT-MD STUDY GROUP

Benoit H. Mulsant ${ }^{1,2,5}$, Tarek K. Rajji ${ }^{1,2,3,5}$, Nathan Herrmann ${ }^{5,9}$, Bruce G. Pollock ${ }^{1,2,5}$, Lillian Lourenco ${ }^{2}$, Daniel M. Blumberger ${ }^{1,2,3,5}$, Christopher R. Bowie ${ }^{1,4}$, Meryl A. Butters ${ }^{6}$, Corinne E. Fischer ${ }^{5,7}$, Alastair Flint ${ }^{5,8}$, Damien Gallagher ${ }^{5,9}$, Angela Golas ${ }^{2,5}$, Ariel Graff ${ }^{1,2,11}$, James L. Kennedy ${ }^{1,5}$, Sanjeev Kumar ${ }^{1,2,3,5}$, Linda Mah ${ }^{5,10}$, Shima Ovaysikia ${ }^{2}$, Mark Rapoport ${ }^{5,9}$, Kevin E. Thorpe ${ }^{12,13}$, Nicolaas P. L. G. Verhoeff $^{11,14}$ and Aristotle N. Voineskos ${ }^{1,2,5}$

${ }^{11}$ Division of Geriatric Psychiatry, Department of Psychiatry, University of Toronto, Toronto, ON, Canada. ${ }^{12}$ Dalla Lana School of Public Health, University of Toronto, Toronto, ON, Canada. ${ }^{13}$ Applied Health Research Centre, Li Ka Shing Knowledge Institute, St. Michael's Hospital, Toronto, ON, Canada. ${ }^{14}$ Department of Psychiatry, Baycrest Health Sciences Centre, Toronto, ON, Canada. 